\title{
The role of D-serine in peripheral tissues
}

\author{
Camila Montesinos Guevara, Ali R. Mani* \\ Division of Medicine, Royal Free Campus, University College London, London, UK
}

* Corresponding author:

Ali R. Mani MD, PhD

Division of Medicine,

Royal Free Campus,

University College London,

Rowland Hill Street,

London, NW3 2PF, UK

Email: a.r.mani@ucl.ac.uk 


\begin{abstract}
A considerable level of D-serine (a free D-amino acid) was discovered, surprisingly, in the mammalian brain in the early 1990's. Since then, D-serine has been considered to be a co-agonist of glutamate at the glycine site of NMDA receptors. D-serine is synthetized by racemization of L-serine in most neural and non-neural cells, and modulates a variety of physiological functions in mammals. In addition to the central nervous system, NMDA receptors have an important function in the modulation of physiological processes in peripheral tissues. Thus, investigations on the functions of D-serine in the peripheral nervous system, as well as the visceral organs, have gained attention in recent years. In this review we summarize the current knowledge on the role of D-serine in the kidneys, skeletal system, skin as well as on the non-adrenergic, non-cholinergic transmission within the autonomic nervous system.
\end{abstract}

Key words: D-serine, serine racemase, NMDA

\title{
1. Introduction
}

Until 1992 it was believed that D-amino acids did not exist in substantial quantities in eukaryotes and that only L-amino acids were important for their function as protein building blocks and metabolic intermediates. However, after Hashimoto et al. (1992) discovered the presence of free Dserine in rat brain, the study of D-amino acids in the body acquired relevance for investigation. In 1999, Wolosker et al. purified a soluble enzyme from rat brain that catalyses the direct racemization of L-serine to D-serine (Wolosker et al., 1999). Further investigations showed that D-serine plays an important role in the central nervous system as an endogenous ligand for the glycine site of glutamate N-Methyl-D-Aspartate (NMDA) receptors (Mothet et al., 2000). Since then, the role of D-serine has been extensively studied in the central nervous system (Gundersen et al., 2015; Bardaweel et al., 2014). In addition to the central nervous system, NMDA receptors have an important function in the modulation of physiological processes in peripheral tissues (Gill and Pulido, 2001). Thus, investigations on the functions of D-serine in the peripheral nervous system, as well as the visceral organs, have gained attention in recent years. In this review we summarize the current knowledge on the role of D-serine in peripheral tissues.

\subsection{Cell expression, synthesis and degradation of D-serine}

D-Serine synthesis is attributed to Serine Racemase (SR), which catalyses the synthesis of D- 
serine from L-serine (Wolosker and Mori, 2012). SR is localized in different tissues of the central nervous system, as well as peripheral tissues (Xia et al., 2004). In the periphery, SR mRNA has been found in the heart, skeletal muscle, kidney, liver (Xia et al., 2004), lower oesophageal sphincter (Ghasemi-Kasman, Dehpour, \& Mani, 2012), corpus cavernosum (Ghasemi et al., 2010), chondrocytes (Takarada, et al., 2008), osteoblasts (Takarada et al., 2012) and skin (Inoue et al., 2014). In addition to performing the racemization reaction, Foltyn, et al. (2005) found that SR is also involved in the elimination of D-Serine through $\alpha-\beta$ elimination activity, generating pyruvate and ammonia. D-serine metabolism is also regulated by allosteric modulators, such as ATP. Foltyn, et al. (2005) found that ATP had moderate effects on the partitioning between racemization of L-serine and $\alpha-\beta$ elimination, favouring $\alpha-\beta$ elimination and stimulating its efficiency 10 -fold. Previous experiments (De Miranda, et al. 2002) revealed that the complex Mg-ATP acts as a physiological cofactor that stimulates 5 to 10 times the rates of racemization and pyruvate production. In general, this data demonstrates the involvement of ATP in modulation of SR function.

In addition to modulation of D-serine level by SR, D-amino acid oxidase (DAAO) is another enzyme that can degrade D-serine in both the central nervous system as well as peripheral tissues. DAAO was first discovered in 1935 by Sir Hans Krebs (Krebs, 1935) whilst performing experiments on the metabolism of amino acids in porcine kidney. Western blot analysis of DAAO has shown high levels of DAAO protein in the cerebellum, however, no DAAO has been detected in the frontal cortex, hippocampus or striatum, suggesting that DAAO may regulate D-serine levels in the cerebellum only (Horio et al., 2011). DAAO protein was also found to be expressed at high concentrations in the kidney of wild type (WT) and SR knockout (SR-KO) mice (Horio et al., 2011), but was not detected in other tissues, such as the lower oesophageal sphincter (Ghasemi-Kasman et al., 2012), corpus cavernosum (Ghasemi et al., 2010), bone cells (Takarada et al., 2012) or mouse liver (Konno et al., 1997). Thus, both SR and DAAO play an important role in the regulation of Dserine concentration in tissues.

\subsection{D-serine and human diseases}

After D-serine was discovered in the rat brain (Hashimoto et al., 1992), further studies investigated its effects in the central nervous system. It was found that neural cells synthesize D-serine from Lserine through SR. SR is abundantly expressed in glial cells, but both neurons and glial cells are involved in D-serine synthesis (Ehmsen et al., 2013). Furthermore, studies revealed that brain D-serine is a physiological co-agonist of glutamate, almost as potent as glycine, which acts on the key neurotransmitter receptor NMDA for its full activation (Mothet et al., 2000). Had D-serine been discovered sooner in the mammalian brain, then the glycine site on the NMDA receptors would probably have been named ' $D$-serine site' instead. NMDA receptors are essential for excitatory synaptic transmission involved in physiological pathways, such as learning and memory, which are 
linked to long-term synaptic plasticity in the hippocampus caused by Long-Term Potentiation (LTP) (Wolosker, et al., 2006). Yang et al. (2003) demonstrated that astrocyte-derived D-serine was required to induce LTP in an NMDA receptor-dependent manner.

NMDA receptor activity is highly regulated, since its over-activation leads to neurotoxicity, as seen in pathological conditions, such as stroke and neurodegenerative diseases (Kemp and McKernan, 2002). Experiments in rat brain cell cultures showed that neuronal D-serine accounts for a significant fraction of NMDA receptor-mediated neurotoxicity in addition to glia contribution with the production of growth factors and regulation of extracellular glycine levels (Kartvelishvily, et al. 2006).

Furthermore, D-serine may play a role in the pathophysiology of neuropsychiatric disorders, such as schizophrenia, which may be linked to NMDA receptor hypo-function (Wolosker et al., 2002). Studies in genetic and pharmacological animal models with decreased D-serine levels have shown that these animals displayed behavioural abnormalities similar to those seen in schizophrenia (Labrie et al., 2012). Moreover, exogenous administration of D-serine and related compounds improved several phenotypes relevant to schizophrenia, which could have positive clinical implications in humans (Labrie, Wong, \& Roder, 2012). Tsai, et al. (1998) performed a clinical trial in Taiwanese schizophrenic patients who received D-serine as adjuvant treatment. The results indicated that those patients who received D-serine treatment, improved positive, negative and cognitive symptoms seen in schizophrenia. In addition, this clinical trial showed that D-serine did not worsen side effects from other antipsychotics, which may be due to its selective action at the NMDA-glycine site. Therefore, D-serine could be considered as a therapeutic approach for schizophrenia, which is different from the dopaminergic approach. However, further clinical trials are required to confirm the efficacy of D-serine therapy. D-serine role in central nervous system disorders also involves chronic epilepsy, in which low D-serine levels may lead to a deficiency in NMDA receptor activation, synaptic plasticity and cognitive function. Thus, the administration of exogenous D-serine may normalise NMDA receptor function and treat cognitive dysfunction seen in epilepsy (Klatte et al., 2013). It has been shown that serum levels of serine enantiomers are higher in patients with depression (Hashimoto et al., 2015) and that D-serine levels are also higher in Alzheimer's disease (Madeira et al., 2015). Therefore, D-serine has the potential to be used clinically as a novel biomarker or treatment for some neuropsychiatric disorders. Although the function of D-serine within the central nervous system has been studied extensively (Bardaweel et al., 2014), details of its role in peripheral organs remain to be uncovered. It is important, given the fact that SR is expressed in peripheral tissues. Thus, any intervention that involves elevation or inhibition of D-serine concentrations in the management of neuropsychiatric conditions, may have unwanted side effects on peripheral organs (e.g. nephrotoxicity). The focus of this review is on our current understanding of the role of D-serine in peripheral tissues. 


\section{D-serine Concentration in the Peripheral Tissues}

Apart from neural tissues, D-serine has been detected in plasma as well as peripheral organs. Horio et al. (2011), studied the contribution of SR in D-serine synthesis in peripheral tissues by comparing Dserine levels in SR-KO and WT mice. Their data showed that D-serine levels in the kidneys, testes and muscles were significantly lower in SR-KO mice compared to WT mice. Whereas, D-serine concentrations in the liver, spleen, pancreas, epididymis, heart, lungs and eyeballs did not differ from those found in SR-KO mice. In addition, serum levels of D-serine were not different between WT and SR-KO mice, suggesting that the presence of D-serine in the blood may not contribute to the different levels of D-serine observed in various peripheral organs (Horio et al., 2011). Thus, other pathways of D-serine production may exist in mammalian tissues; however, this is an area that needs further investigation.

Although it has been found that SR is the main catalytic pathway of D-serine in the brain, other sources of $\mathrm{D}$-serine production could be possible within the brain or they could also originate from the periphery. In addition it is important to consider that $\mathrm{D}$-serine poorly diffuses through the blood-brain barrier as its transporters saturate at certain D-serine concentration in the blood (Pernot et al., 2012). Despite this, Pernot et al. (2012) demonstrated that D-serine administration almost doubled intracellular and extracellular concentrations in the brain. Thus this higher concentration may increase NMDA activation and consequently D-serine from the periphery or exogenous D-serine administration could be involved in modulation of NMDA receptor within the brain.

Since both D-serine and glycine are co-agonists for NMDA receptors, the relative concentration of these two amino acids is important for the contribution of D-serine in modulation of NMDA receptors. Horio et al., (2011) quantified the concentrations of D-serine and glycine in different peripheral organs as well as serum in mice. They showed that the relative concentration of glycine/Dserine ratio is $\sim 1000,300,160$ and 110 in serum, kidney, liver and heart, respectively. This indicates that solid organs have higher relative concentration of D-serine than extracellular fluid.

Furthermore, experimental models used for the study of D-serine in tissues are mainly performed in SR-KO mice. However, other experimental models such as LEA/SEN rats have been studied to understand the role of DAAO in mammals. These rats (LEA/SEN rats) are natural mutants that lack DAAO (Hamase et al., 2010) and exhibit high plasma and urinary D-serine levels. Therefore, these rats have the potential to be used for the study of the role of D-serine in the peripheral tissues (Miyoshi et al., 2011). The function of D-serine in peripheral organs has been a subject of research for the last decade and most of these studies have used either pharmacologic or molecular biology approaches to uncover the function of D-serine in peripheral organs. The current knowledge on Dserine function is described in the following sections. 


\subsection{D-serine in the kidneys}

Evidence for the involvement of D-serine in renal physiology is based on experimental studies in rats (Ma, et al., 2008) and mice (Sasabe et al., 2014). Sasabe et al. (2014) reported that only a minor fraction of $\mathrm{D}$-serine is reabsorbed by mouse nephrons, while most L-serine is reabsorbed in physiological conditions. The same investigators showed that DAAO is mainly expressed in proximal epithelial cells, and that inactivation of this enzyme has a significant effect on plasma levels of Dserine in mice. Thus, the kidneys play an important role in the degradation, as well as excretion of circulating D-serine.

In addition to excretion and degradation, there is evidence to suggest that D-serine is synthetized in rat kidneys. SR is predominantly expressed in rat renal pelvis and medulla. Immunostaining studies demonstrated that the intrapelvic expression of SR was in the smooth muscle layers of the renal pelvis and, to a lesser extent, in the uroepithelial cells (Ma, et al., 2008). Furthermore, the existence of Dserine derived from SR, and its interaction with NMDA receptors may play a role in the reno-renal reflex. It was reported that GluN1 (a NMDA receptor subunit) is expressed in afferent nerves in the renal pelvis and, particularly, in the fibrous structures between the uroepithelial and smooth muscle layers (Ma, et al., 2008). Ma et al. (2008) demonstrated that NMDA receptors are involved in the renal reflex control of body fluid, since the blockade of NMDA receptor by MK-801 caused a decrease in urine output and sodium excretion. Furthermore, experiments showed that intra-pelvic administration of D-serine caused an increase in afferent renal nerve activity and substance P release, with no effects on systemic arterial pressure (Ma, et al., 2008). Overall, this study provided evidence that, under physiological conditions, the increase of intra-pelvic pressure causes activation of the Dserine/NMDA pathway, which in turn acts as mechanoreceptor to allow the influx of $\mathrm{Ca}^{++}$and substance $\mathrm{P}$ release that induce arterial sensory nerve activation (Fig. 1).

Two decades before the discovery of endogenous D-serine in mammals, it was known that injection of exogenous D-serine negatively affects the anatomy and physiology of the kidneys inducing nephrotoxicity leading to extensive necrosis of the proximal tubules (Ganote et al., 1974). Although the mechanism of such nephrotoxicity is not well understood, it was hypothesized that the metabolism of D-serine in the proximal tubules may produce a toxic byproduct in the proximal tubular cells. As discussed above, renal DAAO activity is detected exclusively in proximal tubules and catalyzes the following reaction:

D-serine $+\mathrm{O}_{2} \rightarrow$ Hydroxypyruvate $+\mathrm{NH}_{3}+\mathrm{H}_{2} \mathrm{O}_{2}$

Since $\mathrm{H}_{2} \mathrm{O}_{2}$ is a byproduct of the DAAO enzyme, the production of reactive oxygen species and tissue injury after administration of D-serine is expected. In order to test the hypothesis that DAAO is 
involved in D-serine nephrotoxicity, Maekawa et al., (2005) used rats that lacked DAAO (LEA/SEN rats) in which urinary markers and volume remained normal after being treated with D-serine. These findings were consistent with those of Williams \& Lock (2005), which showed that pre-treatment of rats with sodium benzoate (a competitive inhibitor of DAAO), resulted in reduction of nephrotoxicity caused by D-serine, in a dose-dependent manner. Although this evidence suggests a role of DAAO, its involvement of $\mathrm{H}_{2} \mathrm{O}_{2}$ /oxidative stress in D-serine-induced nephrotoxicity has been questioned (Orozco-Ibarra et al., 2007). Experiments indicated that, although D-serine injections lead to renal tubular necrosis in rats, protein carbonyl content and malondialdehyde (a lipid peroxidation byproduct) remained unchanged (Orozco-Ibarra et al., 2007). Therefore, understanding the molecular mechanism of D-serine nephrotoxicity awaits further investigation.

Recent data about the role of endogenous D-serine in the pathophysiology of renal insufficiency comes from a study in a rat model of lipopolysaccharide (LPS)-induced renal injury (Lin, et al., 2015). It was demonstrated that LPS interacts with Toll-like receptors to induce the release of cytokines, such as interleukin-1 (IL-1). IL-1 leads to the upregulation of SR and the GluN1 subunit of NMDA receptors in the kidneys. This upregulation appears to cause the over-activation of NMDA receptors in tubular cells via D-serine, which seems to induce vasoconstriction, causing poor renal perfusion and ultrafiltration. However, these effects were ameliorated by the inhibition of NMDA receptors with an MK-801 blocker, which also attenuated tubular cell damage in vivo and in vitro (Fig. 2). Likewise, IL-1 receptor antagonism could reduce LPS-induced cytotoxicity and abrogate LPS-induced GluN1 activation, SR upregulation and D-serine secretion. Thus, the results of this study indicated that NMDA receptor hyper-function via cytokine action contributes to LPS-induced renal insufficiency, and that the blockage of these receptors may be useful for the treatment of sepsis-associated renal failure (Lin, et al., 2015).

\subsection{D-serine in the enteric nervous system}

D-serine activity in other peripheral systems, such as the enteric nervous system, has also been studied. Ghasemi-Kasman et al. (2012) investigated the expression of SR and the effects of D-serine in the Non-Adrenergic, Non-Cholinergic (NANC) contraction of the isolated lower oesophageal sphincter (LES) in rats. SR mRNA was detectable using RT-PCR, and SR protein presence was confirmed by Western blot analysis. However, further studies are required to determine the cell type that expresses SR in rat LES. Furthermore, DAAO was not detected in rat LES using RT-PCR, which leads to consider other ways of D-serine degradation.

In organ bath studies of LES strips incubated with D-serine, it was demonstrated that NANC contraction increased after applying electrical field stimulation to the LES strips. This effect was reduced by an NMDA receptor inhibitor (MK-801), which shows that D-serine effects are mediated by NMDA receptor (Ghasemi-Kasman, Dehpour, \& Mani 2012), (Fig. 3). 
Likewise, incubation of isolated LES strips with L-serine caused the same effects as D-serine in NANC contraction. This effect was prevented by SR inhibition. Nevertheless, SR inhibition had no significant effects on NANC contraction in response to electrical field stimulation, which indicates that D-serine may not be compulsory for NMDA receptor-dependent neurotransmission in rat LES. Thus, glycine may act as co-agonist of glutamate at the NMDA receptor in rat LES when D-serine is absent. Results from this study also showed that incubation with D-serine did not affect NANC relaxation, but further studies are required to indicate if D-serine is able to enhance the release of substance P in LES during contraction (Ghasemi-Kasman, Dehpour, \& Mani, 2012).

\subsection{D-serine in corpus cavernosum}

Regarding studies in other organs, it is known that NANC relaxation of corpus cavernosum plays a crucial role in penile tumescence (Rajfer, et al., 1992). Although it was shown that NMDA receptors are expressed in corpus cavernosum (Gonzalez-Cadavid, et al., 2000), the role of D-serine was not studied in corporal relaxation until recently (Ghasemi et al., 2010). Organ bath studies showed that neurogenic relaxation increased when incubating rat corpus cavernosum with D-serine, but not Lserine. RT-PCR and Western blot analysis also provided evidence for the expression of SR in rat corpus cavernosum. Immuno-gold electron microscopy demonstrated that SR is localized in the cavernosal nerve membrane in rats (probably the Schwann cells) (Ghasemi et al., 2010). However, there has been no evidence of DAAO expression in corporal tissues and, therefore, further studies on D-serine degradation are required.

Ghasemi et al. (2010) also indicated that the effect of D-serine is probably mediated by NMDA receptor activation, since various NMDA receptor antagonists might prevent the effect of $\mathrm{D}$-serine on corporal relaxation. It was also postulated that $\mathrm{D}$-serine modulates corporal relaxation by enhancing the release of nitric oxide (NO) from NANC neurons (Fig. 4). The role of D-serine/NMDA receptor in modulating neurogenic relaxation of rat corpus cavernosum may be important for the treatment of impotence and may explain the therapeutic value of ketamine (an NMDA receptor antagonist) in the clinical management of priapism (Uriioersrty and Linda, 1985).

\subsection{D-serine in cartilage and bone physiology}

Takarada, et al., (2008) investigated the role of SR in the regulation of chondrogenic differentiation in tibia cartilage of neonatal rats. They showed that all chondrocytic layers, including proliferating, pre-hypertrophic and hypertrophic chondrocytes, expressed SR. Moreover, chondrogenic ATDC5 cells were cultured to assess the effect of SR overexpression in cartilage. ATDC5 cells are used as in vitro model of chondrocyte mineralisation, which allows the study of 
endochondral ossification (Newton et al., 2012). The experiment in these cells showed that overexpression of SR could negatively regulate cellular differentiation and maturation through the inhibition of SOX9 transcriptional activity (Fig. 5). SOX9 is a transcription factor essential in mesenchymal progenitor cells for their differentiation into chondrocytes. SOX9 is also important for the inhibition of chondrocyte hypertrophy (Ikegami et al., 2011).

In another study Takarada et al., (2009) reported that prolonged exposure to D-serine of cultured rat costal chondrocytes leads to a significant decrease in alkaline phosphatase activity, as well as chondrocytes maturation. More importantly this report provided evidence that D-serine acts as an antagonist of glycine at the NMDA receptor in the cartilage (which is composed of GluN1 and GluN3A subunits). This report is particularly important as it gives a different view of D-serine/glycine interaction at the NMDA receptor in the cartilage, in comparison with the nervous system (Fig. 5). Findings of D-serine's action in chondrocytes led to further investigations on the role of D-serine in bone cell biology (Takarada et al., 2012). Cultured rat calvarial osteoblasts and cultured mouse tibial and femoral osteoclasts were studied. It was found that SR mRNA is only expressed in osteoblasts, but not in osteoclasts, and DAAO mRNA is not present in either of these bone cell cultures. Likewise, it was found that D-serine is present in osteoblasts, but not in osteoclasts. A sustained exposure to Dserine had no effects in osteoblastogenesis, since it did not affect alkaline phosphatase activity and $\mathrm{Ca}^{++}$accumulation in the osteoblasts. However, it affected osteoclastogenesis by inhibiting the differentiation and maturation of cultured osteoclasts in a concentration dependent manner. These findings gave rise to the idea that D-serine may be released from osteoblasts to suppress cellular differentiation of adjacent osteoclasts through inhibition of extracellular L-serine uptake in a competitive manner (Fig. 6).

\subsection{D-serine in the skin}

The study of D-serine synthesis and its influence on the physiological function of the skin has been performed in SR-KO and wild type mice (Inoue et al., 2014). SR protein expression was detected by immunoblotting. SR protein was found to be present in the epidermis of wild type mice, but not in the epidermis of SR-KO mice. Moreover, SR immune-reactivity was identified only in the granular and cornified layers of wild type mice epidermis.

The synthesis of D-serine from L-serine in keratinocytes was also identified from cultures. Results showed that D-serine concentrations were higher in wild type mice than in SR-KO mice, while L-serine concentrations remained almost unchanged. In addition, the concentration ratio of Dserine to total serine was significantly higher in wild type cells than in SR-KO keratinocytes, regardless the addition of L-serine (Inoue et al., 2014). 
Furthermore, the expression levels of mRNAs and proteins involved in the differentiation of keratinocytes, such as involucrin, TGase 3 and K10, were examined by RT-PCR and immunohistochemistry. The results showed that expression of all these markers was significantly higher in SR-KO mice than in wild type mice. These findings imply that SR is involved in the terminal differentiation of keratinocytes. Moreover, the effects of SR deletion in the barrier function of the skin were determined by an assay of barrier recovery. This demonstrated that SR-KO mice exhibited lower recovery rates than wild type mice after type stripping. Therefore, SR is important for the formation of the epidermal barrier in mice. No gross histological abnormality in the skin was found in SR-KO mice after haematoxylin-eosin staining (Inoue et al., 2014). In summary, SR and Dserine are required for the differentiation and maintenance of the physiological function of the skin (Fig. 7).

\subsection{D-serine in the liver}

Although the expression of serine racemase has been demonstrated at both mRNA and protein levels in rat liver, its function in hepatic physiology has remained unclear (Ghasemi et al., 2010; Ghasemi-Kasman, Dehpour, \& Mani, 2012). Horio et al., (2011) reported that D-serine is detectable in mouse liver homogenates, and that levels of D-serine in liver samples are not significantly different when SR-KO mice are compared with WT animals. Since mice do not express DAAO in their liver (Konno et al., 1997), this report suggests that other pathways of D-serine production/degradation may exist in mouse liver. In addition, it is not known which cell type (i.e. hepatocytes, hepatic stellate cells, Kupffer cells or neural cells) is involved in D-serine production or metabolism in the mammalian liver. Recent studies have illustrated a role for NMDA receptors in cell growth in hepatocyte-derived cancer cells lines, such as HepG2 cells (Yamaguchi et al., 2013). For instance, Yamaguchi reported that functional NMDA receptors are expressed in hepatocellular carcinomas and that the FOXO (forkhead box, class O) pathway is involved in the growth inhibitory effects of MK801 (an NMDA receptor blocker) in culture. FOXO pathway plays a role in the regulation of cancer cell growth and it is known as a tumour suppressor. In the liver, FOXO pathway is involved in cell proliferation and metabolism (Yamaguchi et al., 2013). It appears that the glutamate/NMDA pathway is involved in hepatocellular carcinoma growth; however, the role of either glutamate co-agonists (i.e. D-serine or glycine) has not been investigated in this context. 


\section{Conclusion}

Recent studies have provided evidence for the contribution of D-serine in the peripheral nervous system, as well as the visceral organs. D-serine has been considered as a target for management of neurological and neuropsychiatric disorders. However, review of the literature suggests potential side effects after manipulation of D-serine concentration in peripheral tissues. Supplementation with exogenous D-serine may induce nephrotoxicity, natriuresis and inhibition of osteochondral development and mineralisation. Likewise, systemic inhibition of D-serine synthesis has the potential to induce gastro-esophageal reflux disease, salt and water retention, impotence and skin lesions. None of these complications have been studied in humans. In consequence, any intervention that involves elevation or inhibition of D-serine concentrations may have unwanted side effects on peripheral organs and should be considered during the design of future clinical trials. Our knowledge on the production, degradation and function of D-serine in the peripheral tissues is scarce and is limited to the kidneys, cartilage, bones, skin and autonomic nervous system in experimental models. The effects of D-serine in cardiovascular and pancreatic tissues still await further investigation. Finally, more studies are also required to shed light on the involvement of D-serine in the physiology of human peripheral organs.

\section{Conflict of interest: None}

\section{Acknowledgment}

The authors thank Ms. A. Scherchen for linguistic editing suggestions. Camila Montesinos Guevara is supported by the Universities of Excellence Scholarship from the Ecuadorian Government.

\section{References}

Bardaweel, S.K., Alzweiri, M., Ishaqat, A.A., 2014. D-Serine in Neurobiology : CNS Neurotransmission and Neuromodulation.

De Miranda, J., Panizzutti, R., Foltyn, V.N., Wolosker, H., 2002. Cofactors of serine racemase that physiologically stimulate the synthesis of the N-methyl-D-aspartate (NMDA) receptor coagonist D-serine. Proc. Natl. Acad. Sci. U. S. A. 99, 14542-14547. doi:10.1073/pnas.222421299

Foltyn, V.N., 2004. Serine Racemase Modulates Intracellular D-Serine Levels through an , Elimination Activity. J. Biol. Chem. 280, 1754-1763. doi:10.1074/jbc.M405726200

Ganote, C.E., Peterson, D.R., Carone, F. a, 1974. The Nature of D-Serine-Induced Nephrotoxicity. Am. J. Pathol. 77, 269-282.

Ghasemi, M., Rezania, F., Lewin, J., Moore, K.P., Mani, A.R., 2010. d-Serine modulates neurogenic 
relaxation in rat corpus cavernosum. Biochem. Pharmacol. 79, 1791-6. doi:10.1016/j.bcp.2010.02.007

Ghasemi-Kasman, M., Dehpour, A.R., Mani, A.R., 2012. D-serine modulates non-adrenergic noncholinergic contraction of lower esophageal sphincter in rats. Eur. J. Pharmacol. 696, 155-60. doi:10.1016/j.ejphar.2012.09.011

Gill, S.S., Pulido, O.M., 2001. Glutamate Receptors in Peripheral Tissues: Current Knowledge, Future Research, and Implications for Toxicology. Toxicol. Pathol. 29, 208-223. doi:10.1080/019262301317052486

Gonzalez-Cadavid, N.F., Ryndin, I., Vernet, D., Magee, T.R., Rajfer, J., 2000. Presence of NMDA receptor subunits in the male lower urogenital tract. J. Androl. 21, 566-78.

Gundersen, V., Storm-Mathisen, J., Bergersen, L.H., 2015. Neuroglial Transmission. Physiol. Rev. 95, 695-726. doi:10.1152/physrev.00024.2014

Hamase, K., Maruyama, R., Zaitsu, K., 2010. Mutant Mice and Rats Lacking d -Amino Acid Oxidase 7, 1450-1458.

Hashimoto, A., Nishikawa, T., Hayashi, T., Fujii, N., Harada, K., Oka, T., Takahashi, K., 1992. The presence of free D-serine in rat brain. FEBS Lett. 296, 33-36. doi:10.1016/00145793(92)80397-Y

Hashimoto, K., Yoshida, T., Ishikawa, M., Fujita, Y., Niitsu, T., Nakazato, M., Watanabe, H., Sasaki, T., Shiina, A., Hashimoto, T., Kanahara, N., Hasegawa, T., Enohara, M., Kimura, A., Iyo, M., 2015. Increased serum levels of serine enantiomers in patients with depression. Acta Neuropsychiatr. 1-6. doi:10.1017/neu.2015.59

Horio, M., Kohno, M., Fujita, Y., Ishima, T., Inoue, R., Mori, H., Hashimoto, K., 2011. Levels of Dserine in the brain and peripheral organs of serine racemase (Srr) knock-out mice. Neurochem. Int. 59, 853-9. doi:10.1016/j.neuint.2011.08.017

Ikegami, D., Akiyama, H., Suzuki, A., Nakamura, T., Nakano, T., 2011. Sox9 sustains chondrocyte survival and hypertrophy in part through Pik3ca-Akt pathways 1519, 1507-1519. doi:10.1242/dev.057802

Inoue, R., Yoshihisa, Y., Tojo, Y., Okamura, C., Yoshida, Y., Kishimoto, J., Luan, X., Watanabe, M., Mizuguchi, M., Nabeshima, Y., Hamase, K., Matsunaga, K., Shimizu, T., Mori, H., 2014. Localization of serine racemase and its role in the skin. J. Invest. Dermatol. 134, 1618-26. doi:10.1038/jid.2014.22

Kartvelishvily, E., Shleper, M., Balan, L., Dumin, E., Wolosker, H., 2006. Neuron-derived D-serine release provides a novel means to activate N-methyl-D-aspartate receptors. J. Biol. Chem. 281, 14151-14162. doi:10.1074/jbc.M512927200

Kemp, J.A., McKernan, R.M., 2002. NMDA receptor pathways as drug targets. Nat. Neurosci. 5, 1039-1042. doi:10.1038/nn936

Klatte, K., Kirschstein, T., Otte, D., Pothmann, L., Muller, L., Tokay, T., Kober, M., Uebachs, M., Zimmer, A., Beck, H., 2013. Impaired D-Serine-Mediated Cotransmission Mediates Cognitive Dysfunction in Epilepsy. J. Neurosci. 33, 13066-13080. doi:10.1523/JNEUROSCI.542312.2013 
Konno, R., Sasaki, M., Asakura, S., Fukui, K., Enami, J., Niwa, a, 1997. D-amino-acid oxidase is not present in the mouse liver. Biochim. Biophys. Acta 1335, 173-81.

Krebs, H.A., 1935. Metabolism of amino-acids: Deamination of amino-acids. Biochem. J. 29, 162044. doi:10.1146/annurev.bi.05.070136.001335

Labrie, V., Wong, A.H.C., Roder, J.C., 2012. Contributions of the d-serine pathway to schizophrenia. Neuropharmacology 62, 1484-1503. doi:10.1016/j.neuropharm.2011.01.030

Lin, C.-S., Hung, S.-F., Huang, H.-S., Ma, M.-C., 2015. Blockade of the N-Methyl-D-Aspartate Glutamate Receptor Ameliorates Lipopolysaccharide-Induced Renal Insufficiency. PLoS One 10, e0132204. doi:10.1371/journal.pone.0132204

Ma, M.-C., Huang, H.-S., Chen, Y.-S., Lee, S.-H., 2008. Mechanosensitive N-methyl-D-aspartate receptors contribute to sensory activation in the rat renal pelvis. Hypertension 52, 938-44. doi:10.1161/HYPERTENSIONAHA.108.114116

Madeira, C., Lourenco, M. V, Vargas-Lopes, C., Suemoto, C.K., Brandão, C.O., Reis, T., Leite, R.E.P., Laks, J., Jacob-Filho, W., Pasqualucci, C. a, Grinberg, L.T., Ferreira, S.T., Panizzutti, R., 2015. d-serine levels in Alzheimer's disease: implications for novel biomarker development. Transl. Psychiatry 5, 1-9. doi:10.1038/tp.2015.52

Maekawa, M., Okamura, T., Kasai, N., Hori, Y., Summer, K.H., Konno, R., 2005. D-amino-acid oxidase is involved in D-serine-induced nephrotoxicity. Chem. Res. Toxicol. 18, 1678-82. doi:10.1021/tx0500326

Miyoshi, Y., Hamase, K., Okamura, T., Konno, R., Kasai, N., Tojo, Y., Zaitsu, K., 2011. Simultaneous two-dimensional HPLC determination of free d-serine and d-alanine in the brain and periphery of mutant rats lacking d-amino-acid oxidase. J. Chromatogr. B Anal. Technol. Biomed. Life Sci. 879, 3184-3189. doi:10.1016/j.jchromb.2010.08.024

Mothet, J.-P., Parent, A.T., Wolosker, H., Brady, R.O., Linden, D.J., Ferris, C.D., Rogawski, M.A., Snyder, S.H., 2000. D-Serine is an endogenous ligand for the glycine site of the N-methyl-Daspartate receptor. Proc. Natl. Acad. Sci. 97, 4926-4931. doi:10.1073/pnas.97.9.4926

Mothet, J.P., Parent, A.T., Wolosker, H., Brady, R.O., Linden, D.J., Ferris, C.D., Rogawski, M.A., Snyder, S.H., 2000. D-serine is an endogenous ligand for the glycine site of the N-methyl-Daspartate receptor. Proc. Natl. Acad. Sci. U. S. A. 97, 4926-4931. doi:10.1073/pnas.97.9.4926

Newton, P.T., Staines, K.A., Spevak, L., Boskey, A.L., Teixeira, C.C., 2012. Chondrogenic ATDC5 cells : An optimised model for rapid and physiological matrix mineralisation 1187-1193. doi:10.3892/ijmm.2012.1114

Orozco-Ibarra, M., Medina-Campos, O.N., Sánchez-González, D.J., Martínez-Martínez, C.M., Floriano-Sánchez, E., Santamaría, A., Ramirez, V., Bobadilla, N. a., Pedraza-Chaverri, J., 2007. Evaluation of oxidative stress in d-serine induced nephrotoxicity. Toxicology 229, 123-135. doi:10.1016/j.tox.2006.10.008

Pernot, P., Maucler, C., Tholance, Y., Vasylieva, N., Debilly, G., 2012. Neurochemistry International D -Serine diffusion through the blood - brain barrier : Effect on D -serine compartmentalization and storage. Neurochem. Int. 60, 837-845. doi:10.1016/j.neuint.2012.03.008

Sasabe, J., Suzuki, M., Miyoshi, Y., Tojo, Y., Okamura, C., Ito, S., Konno, R., Mita, M., Hamase, K., Aiso, S., 2014. Ischemic acute kidney injury perturbs homeostasis of serine enantiomers in the 
body fluid in mice: early detection of renal dysfunction using the ratio of serine enantiomers. PLoS One 9, e86504. doi:10.1371/journal.pone.0086504

Takarada, T., Hinoi, E., Takahata, Y., Yoneda, Y., 2008. Serine racemase suppresses chondrogenic differentiation in cartilage in a Sox9-dependent manner. J. Cell. Physiol. 215, 320-8. doi:10.1002/jcp.21310

Takarada, T., Takahata, Y., Iemata, M., Hinoi, E., Uno, K., Hirai, T., Yamamoto, T., Yoneda, Y., 2009. Interference with cellular differentiation by D-serine through antagonism at N-methyl-Daspartate receptors composed of NR1 and NR3A subunits in chondrocytes. J. Cell. Physiol. 220, 756-764. doi:10.1002/jcp.21821

Takarada, T., Takarada-Iemata, M., Takahata, Y., Yamada, D., Yamamoto, T., Nakamura, Y., Hinoi, E., Yoneda, Y., 2012. Osteoclastogenesis is negatively regulated by D-serine produced by osteoblasts. J. Cell. Physiol. 227, 3477-3487. doi:10.1002/jcp.24048

Tsai, G., Yang, P., Chung, L.C., Lange, N., Coyle, J.T., 1998. D-serine added to antipsychotics for the treatment of schizophrenia. Biol. Psychiatry 44, 1081-1089. doi:10.1016/S0006-3223(98)002790

Uriioersrty, L.L., Linda, L., 1985. Thoughts on Protamine Toxicity Ketamine for Treatment of Priapism Aminophylline Antagonism of the Residual Effects of Fentanyl Anesthesia Myoclonus after Etomidate Anesthesia 1033-1034.

Williams, R.E., Lock, E. a., 2005. Sodium benzoate attenuates d-serine induced nephrotoxicity in the rat. Toxicology 207, 35-48. doi:10.1016/j.tox.2004.08.008

Wolosker, H., 2006. P ERSPECTIVE D -Serine Regulation of NMDA Receptor Activity. Gene Expr. 10-12. doi:10.1126/stke.3562006pe41

Wolosker, H., Mori, H., 2012. Serine racemase: an unconventional enzyme for an unconventional transmitter. Amino Acids 43, 1895-904. doi:10.1007/s00726-012-1370-3

Wolosker, H., Panizzutti, R., Miranda, J.D., 2002. Neurobiology through the looking-glass: D-serine as a new glial-derived transmitter. Neurochem. Int. 41, 327-332. doi:10.1016/S01970186(02)00055-4

Wolosker, H., Sheth, K.N., Takahashi, M., Mothet, J.P., Brady, R.O., Ferris, C.D., Snyder, S.H., 1999. Purification of serine racemase: biosynthesis of the neuromodulator D-serine. Proc. Natl. Acad. Sci. U. S. A. 96, 721-5.

Xia, M., Liu, Y., Figueroa, D.J., Chiu, C.-S., Wei, N., Lawlor, A.-M., Lu, P., Sur, C., Koblan, K.S., Connolly, T.M., 2004. Characterization and localization of a human serine racemase. Brain Res. Mol. Brain Res. 125, 96-104. doi:10.1016/j.molbrainres.2004.03.007

Yamaguchi, F., Hirata, Y., Akram, H., Kamitori, K., Dong, Y., Sui, L., Tokuda, M., 2013. FOXO/TXNIP pathway is involved in the suppression of hepatocellular carcinoma growth by glutamate antagonist MK-801. BMC Cancer 13, 468. doi:10.1186/1471-2407-13-468

Yang, Y., Ge, W., Chen, Y., Zhang, Z., Shen, W., Wu, C., Poo, M., Duan, S., 2003. Contribution of astrocytes to hippocampal long-term potentiation through release of D-serine. Proc. Natl. Acad. Sci. U. S. A. 100, 15194-15199. doi:10.1073/pnas.2431073100 


\section{Figure legends}

Figure 1. Contribution of D-serine in the reno-renal reflex: Increased intra-pelvic pressure causes activation of NMDA receptors and the release of substance P (SP). SP can stimulate renal afferent nerves leading to a natriuretic response. Glu: Glutamate; SR: Serine Racemase; NMDAr: NMDA receptor; *: Unknown carriers. **: Unknown source.

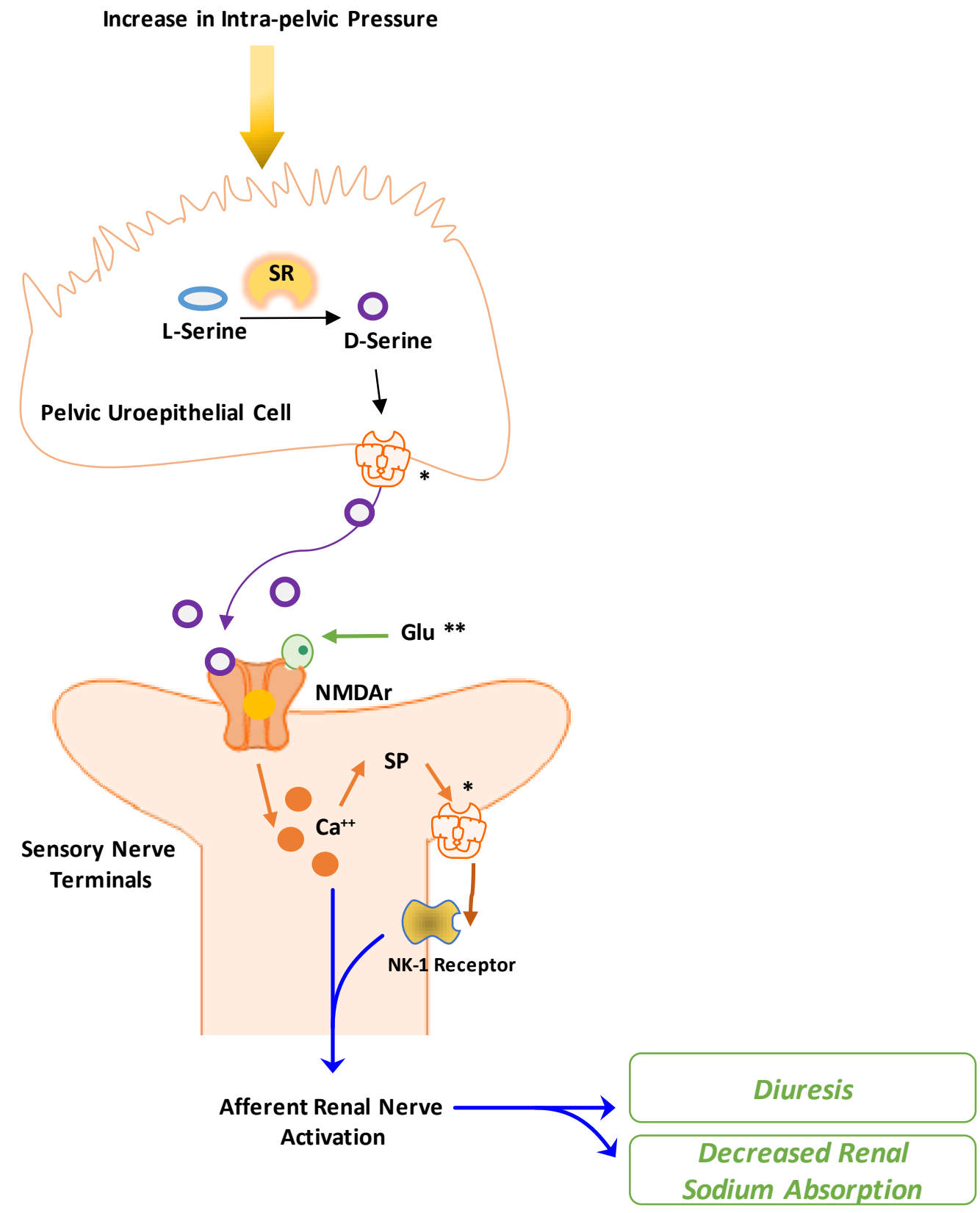


Figure 2. Renal dysfunction caused by endogenous D-serine during systemic inflammation (endotoxemia). Lipopolysaccharide (LPS) acts on Toll-like receptors (TLR4) and leads to the release of interleukin-1 $\beta$ from tubular cells. This, in turn activates Serine Racemase (SR) that catalyzes the conversion of L-serine into D-serine, and also increases the expression of NMDA receptors. NMDA receptor activation induces renal vasoconstriction, hypo-perfusion and tubular damage through the release of intracellular calcium. LPS: Lipopolysaccharide; TLR4: Toll-like receptor 4; IL-1 $\beta$ : Inter Leukin-1ß; IL-1R: Inter Leukin-1 receptor; SR: Serine Racemase; NMDAr: NMDA receptor. Adapted from Lin et al., 2015.

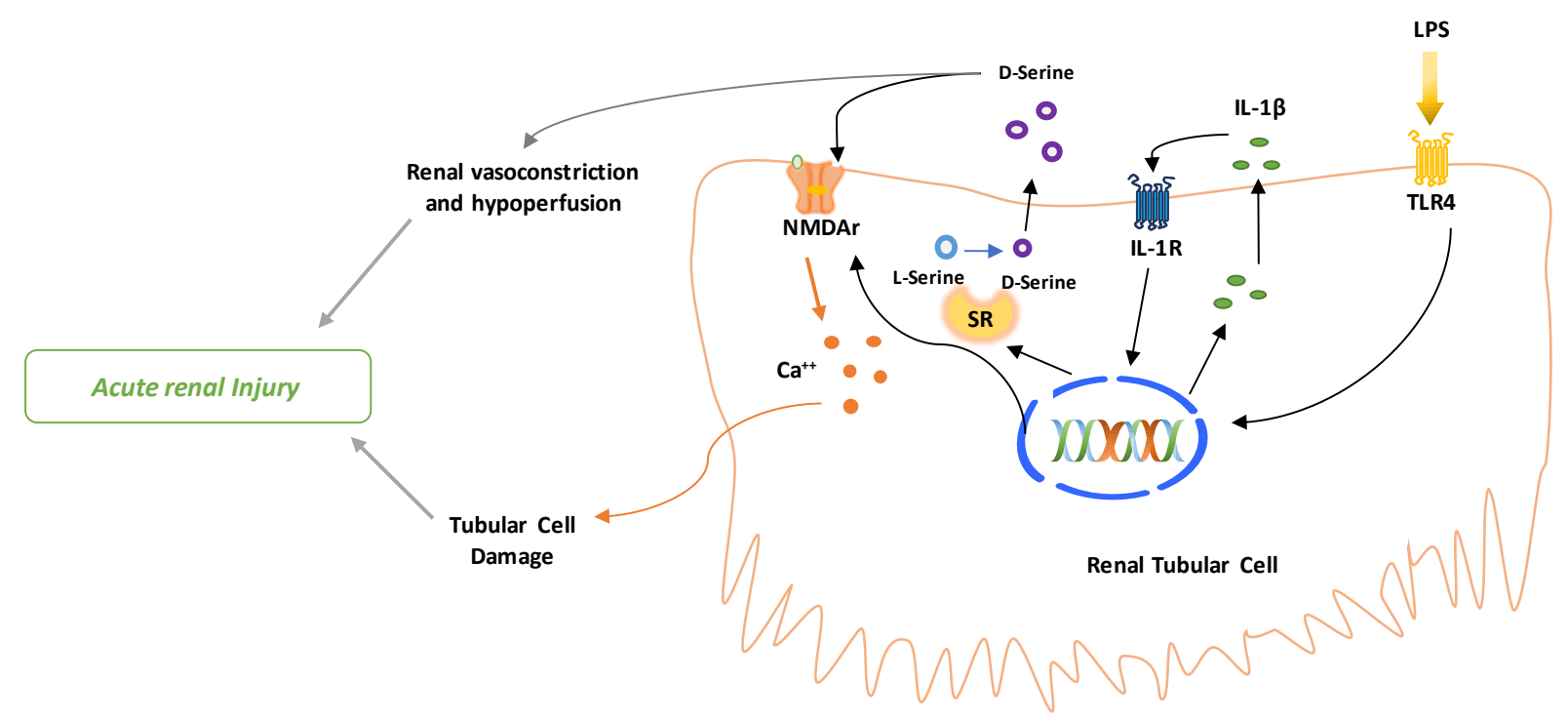


Figure 3. Hypothetical illustration of the role of D-serine/NMDA receptor pathway during contraction of the lower oesophageal sphincter. D-serine modulates NMDA receptors, which can stimulate the enteric neurons to release contractile neurotransmitters such as tachykinins (substance $\mathrm{P}$ or neurokinin). EFS: Electrical Field Stimulation; Glu: Glutamate; SR: Serine Racemase; NMDAr: NMDA receptor; SP: Substance P; *: Unknown carrier, **: Contribution of Neurokinin or SP has been suggested but not shown yet.

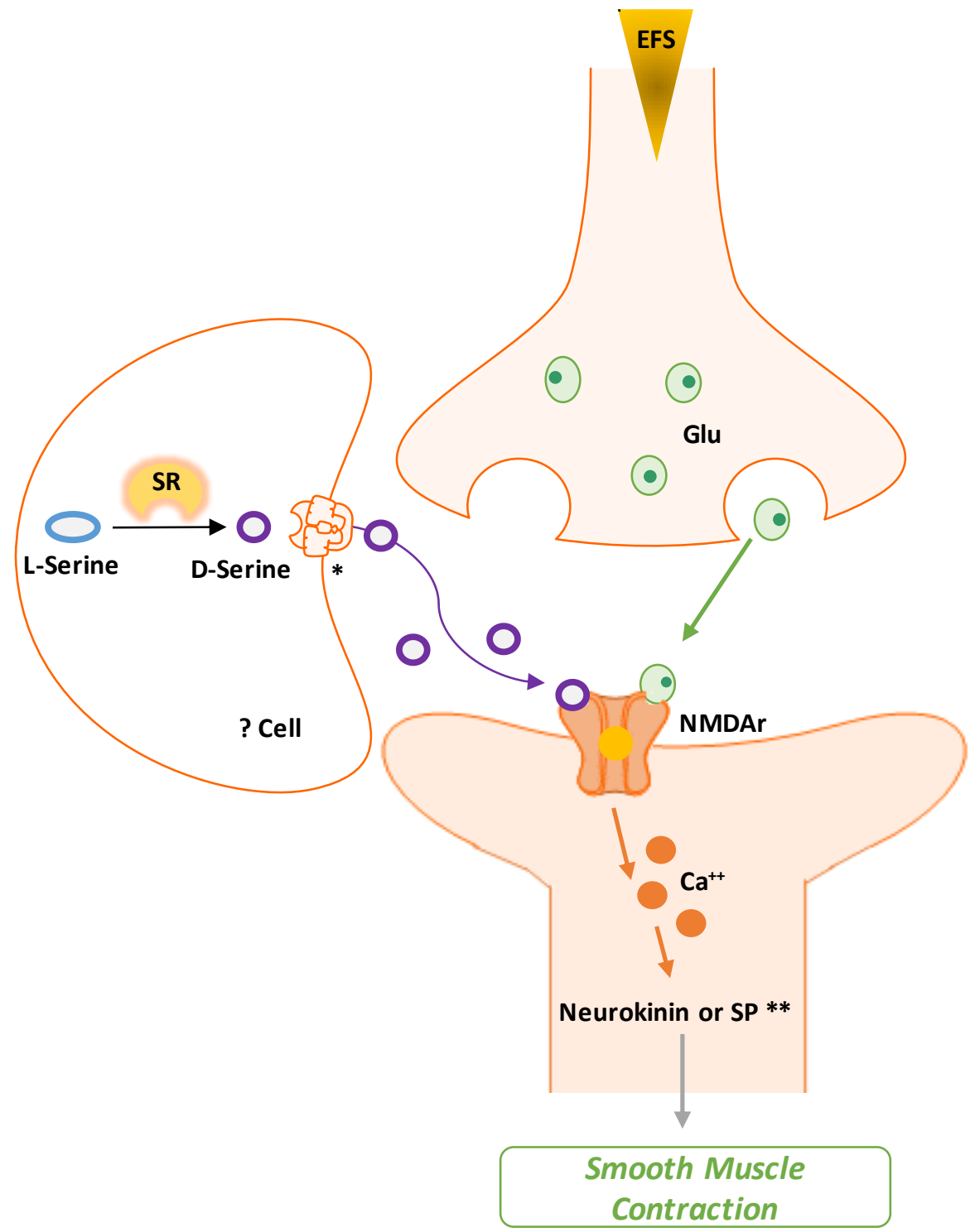


Figure 4. A graphic representation of the neurogenic relaxation of corpus cavernosum induced by DSerine released from glial cells located in close proximity to nerves. D-serine and Glu activate NMDA receptor on postsynaptic nerve. This activation causes $\mathrm{Ca}^{++}$release and consequently the activation of neuronal NO synthase (nNOS) that converts L-arginine to NO. NO is the final mediator for the relaxation of corporal tissue. Glu: Glutamate; SR: Serine Racemase; NMDAr: NMDA receptor; nNOS: Neuronal Nitric Oxide Synthase; NO: Nitric Oxide. *: Unknown carrier.

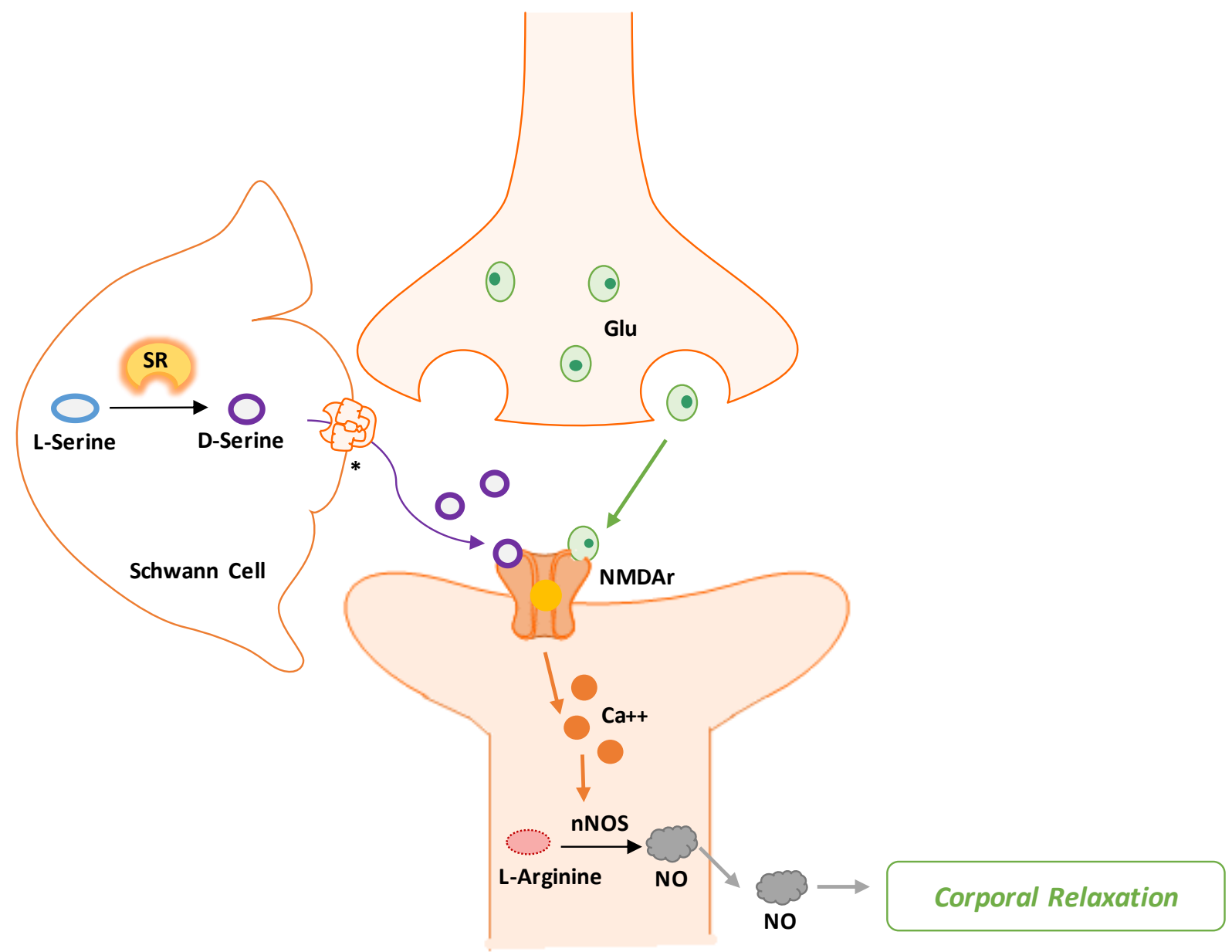


Figure 5. A schematic view of the mechanisms by which intracellular D-Serine leads to inhibition of chondral differentiation, maturation and delayed mineralization. After D-serine is released from chondrocytes, it activates NMDA receptor. The activation of NMDA receptor leads to the interference with Runx2 transcriptional activity and the stimulation of proteosomal degradation of SOX9, an essential protein for chondrocytic differentiation. Glu: Glutamate; Gly: Glycine; SR: Serine Racemase; NMDAr: NMDA receptor. *: Unknown carrier.

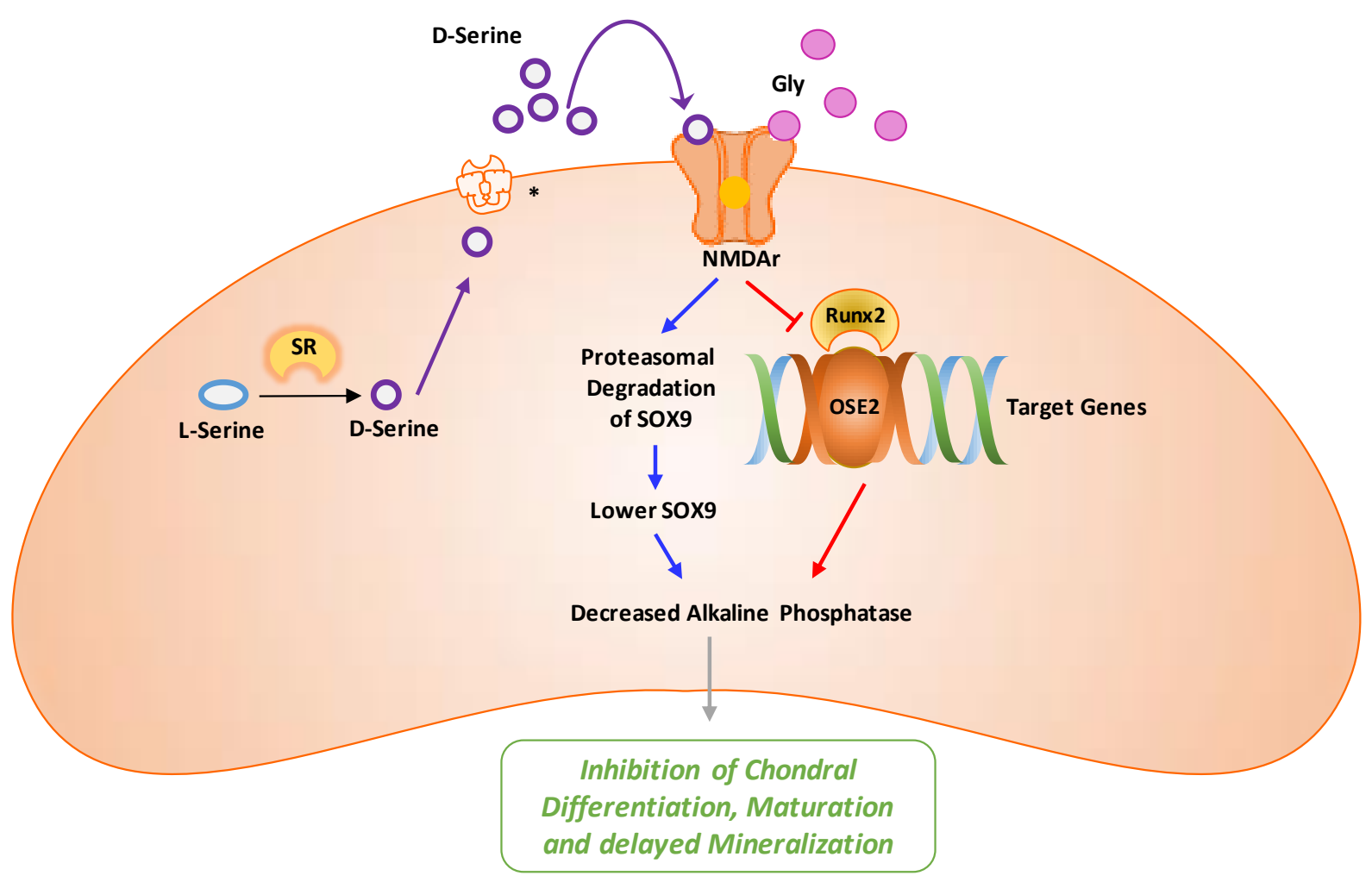


Figure 6. Illustration of the inhibition of osteoclastogenesis by D-serine. After being synthesized in osteoblasts, D-serine is exported to the extracellular space where it prevents the incorporation of Lserine mediated by $\mathrm{ATB}_{0+}$ and ASCT2 receptors. This process leads to the suppression of osteoclastogenesis mediated by L-serine, which is normally coupled to RANKL/RANK-induced osteoclastogenesis pathway. SR: Serine Racemase; *: Unknown carrier. Adapted from Takarada et al., 2012.

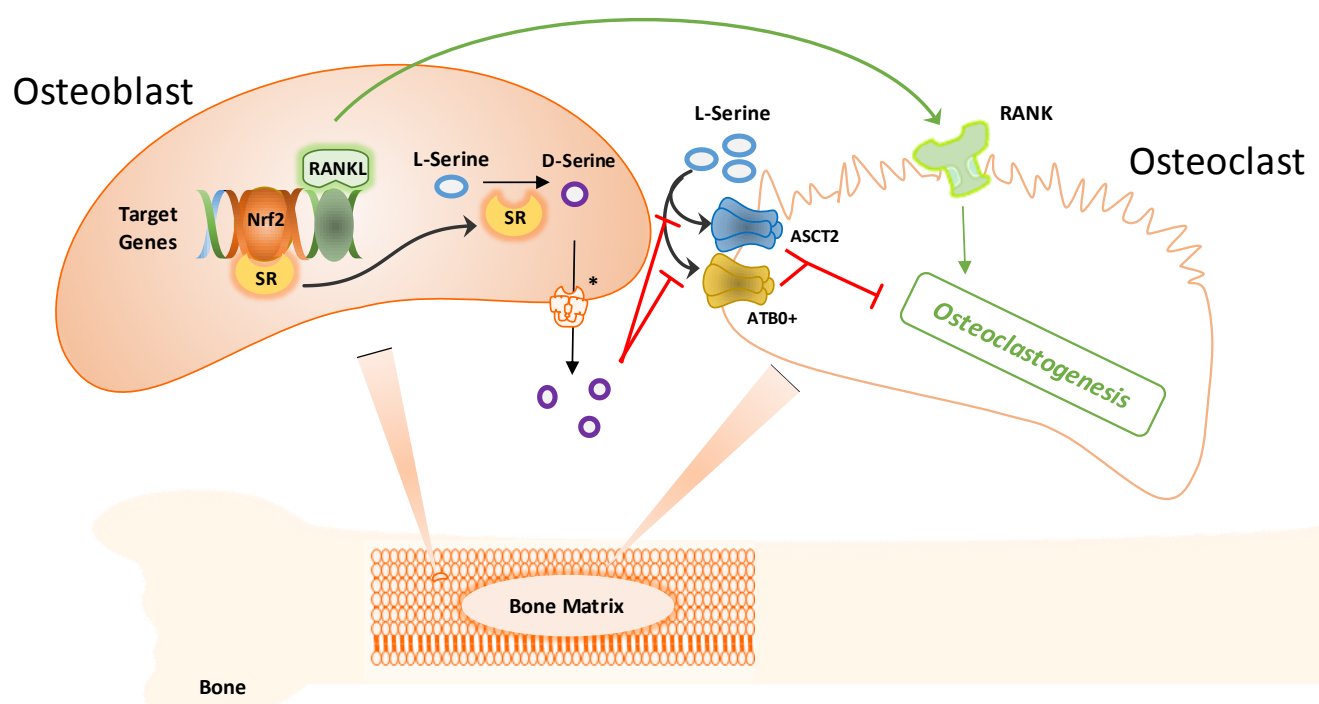


Figure 7. The effects of D-serine on epithelial keratinocytes differentiation and skin barrier formation. After being synthetized by SR, D-serine is released from granular and cornified keratinocytes, where it activates NMDA receptors in conjunction with glutamate (Glu). Afterwards $\mathrm{Ca}^{++}$is released, which increases the expression of markers of differentiation and contributes to the formation of the epithelial barrier. Glu: Glutamate; SR: Serine Racemase; NMDAr: NMDA receptor; KCs: Keratinocytes. *: Unknown carrier.

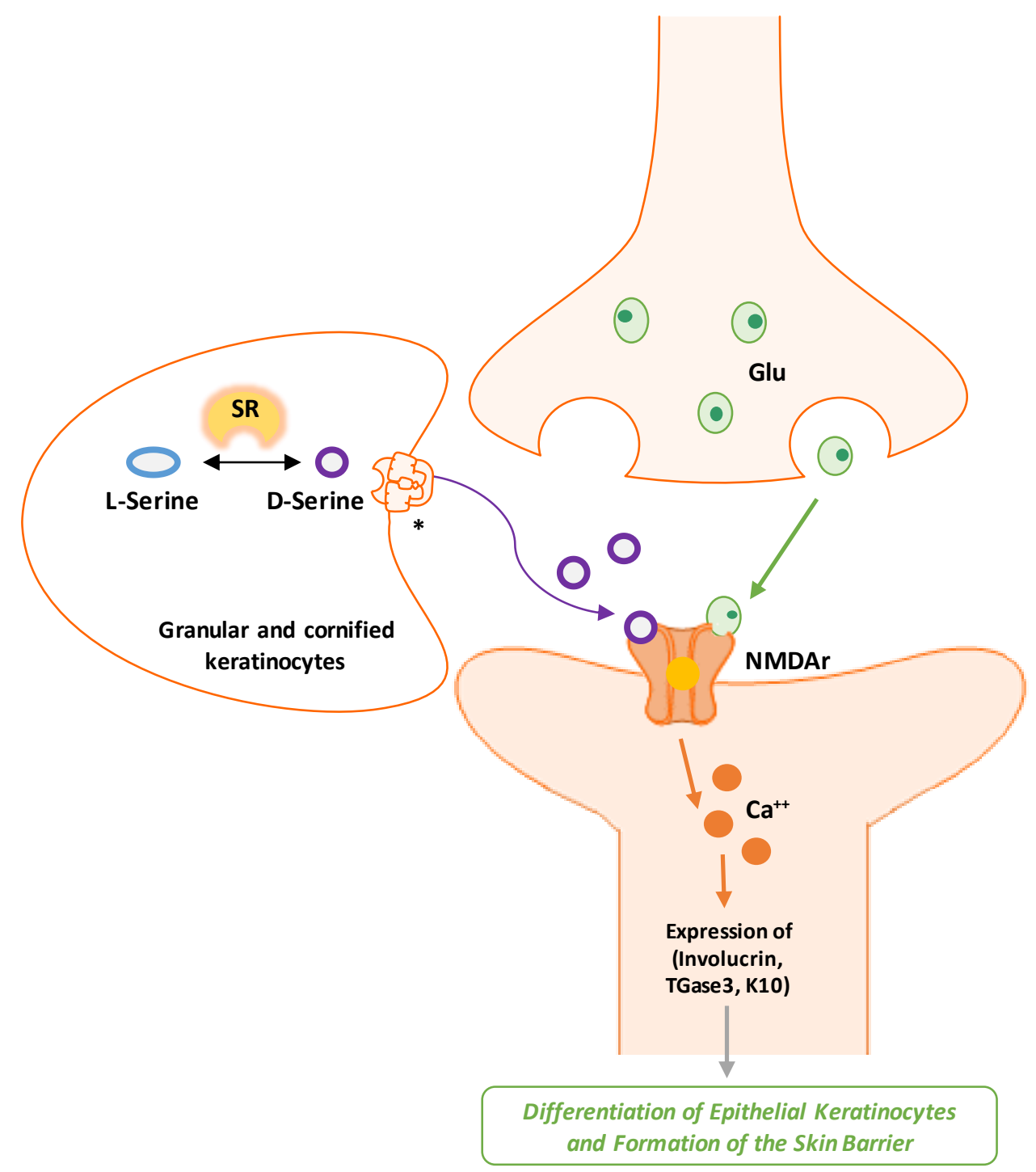

